\title{
Improved Hessian Curvature for Fingerprint Analysis
}

\author{
Shan Juan Xie ${ }^{1}$, Yanshuang Zhang ${ }^{2}$ \\ Hangzhou Normal University, Institute of Remote Sensing and Earth Science, Hangzhou, China ${ }^{1}$ \\ Zhejiang Gongs hang University Hangzhou College of Commerce, Hangzhou, China ${ }^{2}$
}

\begin{abstract}
Curvature analysis is important to the fingerprint image which is constructed by a group of regular ridgelines. Five kinds of curvatures constructed by the eigenvalues are tested, including the Gaussian curvature, Mean curvature, Laplacian curvature, Hessian curvature and our improved Hessian curvature. Researching on these five kinds of curvature, we find the relative advantages of our improved Hessian curvature for fingerprint curves. A measure for fingerprint reference point determination based on the eigenvalues of the covariance matrix of the ridges. Fingerprint reference points are determined by combining the global and local proposed curvature properties of ridges by using multi-resolution levels. The performance of the proposed one is verified through simulations and its analysis.
\end{abstract}

Keywords: fingerprint, reference point, curvature, multi-resolution

\section{INTRODUCTION}

Automatic fingerprint identification system (AFIS) is one of the most popular and reliable technologies for automatic biometric identification. An ideal fingerprint identification method should tolerate changes in the fingerprint input by its orientations, translations, elastic distortions and noises. Since reference points regardless of types of fingerprint images are relatively robust to various orientations, elastic distortions and noises, the fingerprint reference point determination is one of the most commonly used processes for the fingerprint matching method [1]. Especially, it is crucial to localize reference points in fingerprint applications such as a classification of fingerprints and their retrieval from large fingerprint databases, because it can greatly reduce the number of the fingerprints to be compared in the verification step.

A fingerprint is constructed by a group of regular ridgelines which can be recognized as a group of curves with different curvatures. Since the reference point is defined as "the point of the maximum curvature on the convex ridge" [2], the curvature-based method is mostly used for reference point localization, such as block region based methods [2, 3, 4] and pixel based methods [5][6][12]. In this paper, a new curvature based algorithm for the determination of fingerprint reference point is proposed to overcome the demerits of previous methods over poor-quality images. In the proposed algorithm, new orientation certainty values obtained from the relationship of eigenvalues of covariance matrix of image blocks are used to determine a unique reference point more precisely for arch-type of fingerprint images and partial fingerprint images. And also, the proposed method was compared with the complex filtering method proposed by A.K. Jain et al. [7][8] on the spatial and frequency domain [9].

Curvature analysis is meaningful to the fingerprint authentication [10]. There are several curvature definitions in image processing. Besides there are four kinds of curvatures constructed by the eigenvalues, which includes
Gaussian curvature, Mean curvature, Laplacian curvature, Hessian curvature. Each curvature definition has its own principles and its own adoptable areas. In the paper, we make series of experiments to compare their characteristics. Researching on these four kinds of curvature, we define a new proposed curvature is adopted for fingerprint image based on the Hessian curvature. The relative advantages of the improved Hessian curvature for fingerprint curves. In the experimental part, we calculate Euclidean distance between the manually-located reference point position and the reference point position located by the proposed method to compare the accuracy with the mentioned methods.

The rest of the paper is organized as follows. Section 2 introduces the mathematic definition of eigenvalues of covariance matrices. Section 3 explains the procedure for the reference point determination. In Section 4 several experiments are designed to compare the properties of the five kinds of curvature methods and the results are compared with some of the well-known reference point determination algorithms. Finally the paper concludes in Section 5.

\section{CURVATURES CONSTRUCTION USING EIGEN VALUES}

The curvature characteristic indicates the change of orientation. There are several curvature definitions in image processing. Four kinds of curvatures are constructed by the eigenvalues, which includes Guassian curvature, Mean curvature, Laplacian curvature, and Hessian curvature. Each curvature definition has its own principles and its own adoptable areas. In the paper, we make series of experiments to compare their characteristics. Researching on these four kinds of curvature, we define a new curvature is adopted for fingerprint image based on the Hessian curvature. The relative advantages of the 
improved Hessian curvature for fingerprint curves. In general, the normal curvature has a maximum value Introduction about differential geometry and eigen value based curvature.

\section{A. FOUR CONVENTIONAL CURVATURES}

A family of second-order nonlinear differential operations that are useful in estimation object curvature. A complete curvature estimation is usually an intelligent combination of simple eigenvalues from the covariance matrix of the second-order differential operation. In turn, these are often built by applying a suitable differential operator to a suitable filtering of the image. Filtering the image controls the quantitative behaviour of the curvature change, while the eigenvalues inform its qualitative behaviour. In principle, the primary first-order differential quantity of an image is the gradient. The gradient of an image is defined as

$$
\nabla G(x, y)=\left\lceil\begin{array}{l}
G_{x}(x, y) \\
G_{y}(x, y)
\end{array}\right]
$$

The intensity gradient $G_{x}, G_{y}$ of each pixel can be calculated using Prewitt, Sobel or Robert operators. In this paper, Sobel operator of $3 \times 3$ windows is used. It has both direction and magnitude which vary at each point. The gradient direction at a point is the direction of steepest ascent at that point. The gradient magnitude is the steepness of that ascent. And the direction is the normal to the level curve at that point.

Just as we use the gradient direction and perpendicular as first order gauge coordinates, we can use the principal directions as the second-order gauge coordinates. The second-order gauge coordinates are also intrinsic to the image surface as the first-order gauge coordinates. They are most useful for detecting edge, ridges, peaks, corners and vertices. For the second-order geometry, the equivalent of the gradient is the matrix of second derivatives or Hessian.

$$
H=\left[\begin{array}{ll}
G_{x x} & G_{x y} \\
G_{y x} & G_{y y}
\end{array}\right]=\left[\begin{array}{ll}
h_{1} & h_{3} \\
h_{3} & h_{2}
\end{array}\right]
$$

Since $G_{x y}=G_{y x}$, this is a real and symmetric matrix, whose determinant is equal to the product of its eigenvalues and is invariant to the selection $\mathrm{x}$ and $\mathrm{y}$. when the selection of $\mathrm{x}$ and $\mathrm{y}$ are proper, it can produce a diagonal matrix whose eigenvalues are the diagonal elements.

$$
\begin{aligned}
& \lambda_{\min }=\frac{\left(h_{1}+h_{2}\right)-\sqrt{\left(h_{1}-h_{2}\right)^{2}+4 h_{3}{ }^{2}}}{2} \\
& \lambda_{\max }=\frac{\left(h_{1}+h_{2}\right)+\sqrt{\left(h_{1}-h_{2}\right)^{2}+4 h_{3}{ }^{2}}}{2}
\end{aligned}
$$

$\lambda_{\max }$ which is the direction of greatest curvature, and a minimum value $\lambda_{\min }$ which is the direction of least curvature. We separate the fingerprint images into four kinds of regions by their curvature properties, ordinary region, reference point region, edge region and noise region. For each kind of regions, there are 500 representational sample regions with the size of $32 * 32$ pixels collected from the database FVC2004_DB1. The ordinary region always shows the uniform curvature between ridges, while the reference point region has the abrupt difference between the ridges since the reference point brings the maximum curvature. And the noise regions always cause large curvature since the noises affect the curvature calculation easily. The edge region also shows large curvature value as the differences exist between the foreground and background. As our purpose of reference point determination, we need to exclude the interferes of noise region and edge region. In order to find an effective curvature calculation, we analysed the existed curvature calculation methods and proposed our adaptive curvature estimation method for accurate fingerprint reference point determination. A complete curvature estimation is usually an intelligent combination of simple eigenvalues from the covariance matrix of the secondorder differential operation. Fig.1 shows the eigenvalue distribution for the four kinds of regions. As shown in the figure, the ordinary region shows the random distribution of the $\lambda_{\max }$ values, while the else regions have the smaller $\lambda_{\max }$ value. For the $\lambda_{\min }$ values, the reference point region shows a larger range than the other regions, especially, for the edge region and noise region, the $\lambda_{\min }$ values are always smaller. The four regions show various eigenvalue distributions that is helpful to distinguish these four regions. And their own eigenvalue properties are also the academic base for the following curvature test.
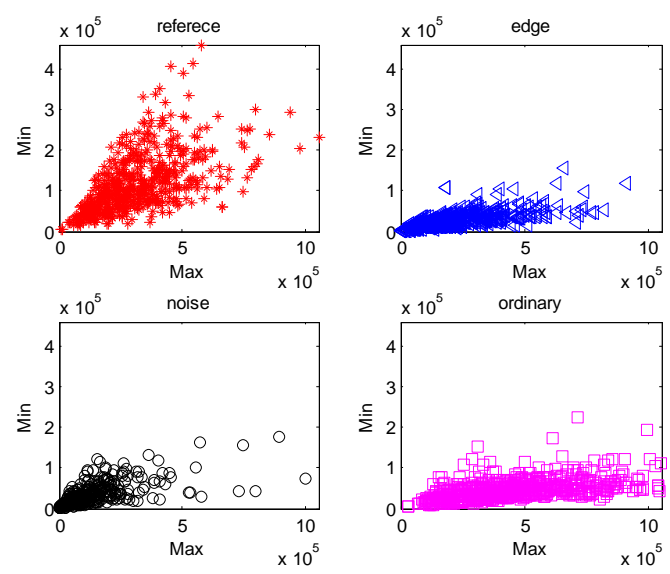

Fig.1. Eigenvalues distribution for four kinds of regions

For the accurate curvature estimation, we researched four kinds of curvaturesdefined based on the relationship of $\lambda_{\min }$ and $\lambda_{\max }$. 


$$
\begin{aligned}
& \text { Hessian curvature : } \operatorname{Cur}_{H}=\lambda_{\min } / \lambda_{\max } \\
& \text { Gaussian curvature: } \operatorname{Cur}_{G}=\lambda_{\min } \cdot \lambda_{\max } \\
& \text { Mean curvature: } \operatorname{Cur}_{\mu}=\left(\lambda_{\min }+\lambda_{\max }\right) / 2 \\
& \text { Laplacian curvature: } \operatorname{Cur}_{L}=\lambda_{\min }+\lambda_{\max }
\end{aligned}
$$

(5) Since the Gaussian curvature ranges from 0 to 1010 , it is hard to separate the four fingerprint regions with their

(6) Gaussian curvatures. Compared with Hessian curvature,

(7) the Mean curvature and Laplacian curvature meet the same case.

The Mean curvature is the average of $\lambda_{\min }$ and $\lambda_{\max }$. It is denoted as $\mathrm{H}$ and equal to the half the trace of $\mathrm{H}$. The Laplacian curvature is simply twice the Mean curvature and is also invariant to rotation. The surface region can be classified according to Mean curvature and Gaussian curvature.

Cur $_{H}$ is the ratio of two eigenvalues of the Hessian matrix of the pixel gradient vector. It indicates the energy centration along on the direction of ridges, which ranges of $[0,1]$. For a large certainty value, ridges and valleys are very clear with good orientation consistency and, as the certainty value decreases, they change irregularly Fig.3 shows the relationship between two eigenvalues and Hessian curvature of the four kinds of representational sample regions. The reference point region shows the larger Hessian curvature values than the else regions, which is range of $[0,1]$. However the else regions, the Hessian curvatures are below 0.5 . It indicates that we can separate the reference point region with the other regions by Hessian curvature values. Not only the differences between the hessian curvature values, if we take attention to the reference point region and the noise region, there are obvious differences between their eigenvalues.

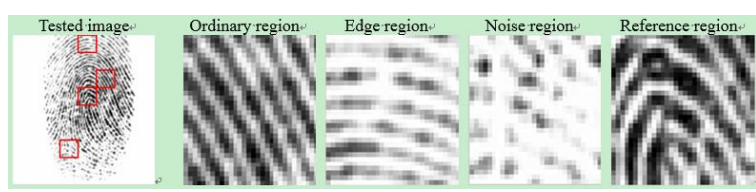

Fig.2. Four kinds of representational sample regions
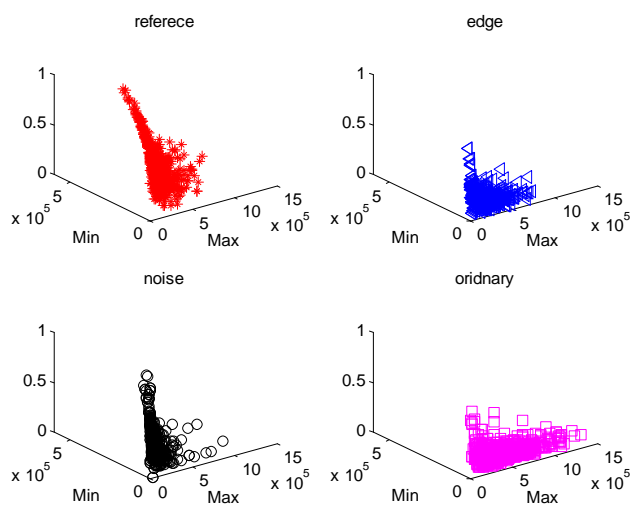

Fig.3. Hessian curvatureanalyses for four kinds of representational sample regions

The same experiments are done for Gaussian curvature, Mean curvature and Laplacian curvature as shown in Fig34. Since the Laplacian curvature is simply twice of the Mean curvature, Laplacian curvature shows the similar curvature relationship as the mean curvature properties shown in Fig.4.
If $\mathrm{Cur}_{G}>0$ which is called Elliptic patches, the curvature in any direction is positive when $\mathrm{Cur}_{M}>0$ and the curvature in any direction is positive when $\mathrm{Cur}_{M}<0$. When $\mathrm{Cur}_{G}<0$, the curvature in some directions are positive and others are negative, which is called Hyperbolic patches

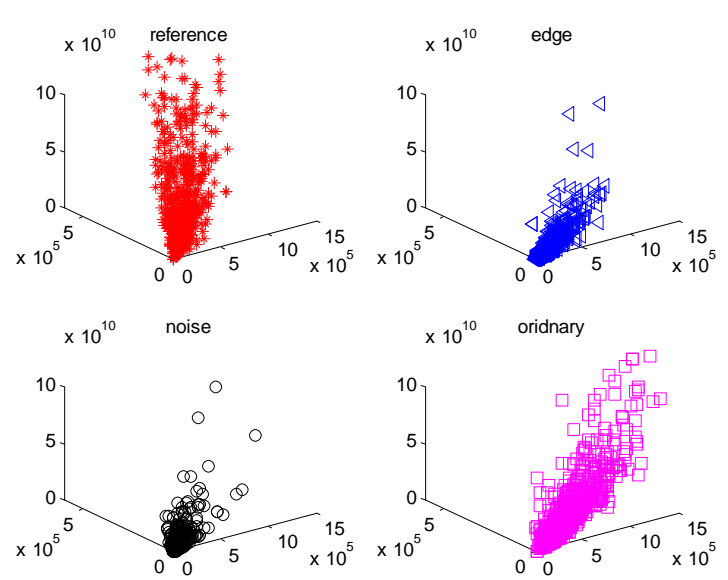

Fig.4. Gaussiancurvatureanalyses for four kinds of representational sample regions
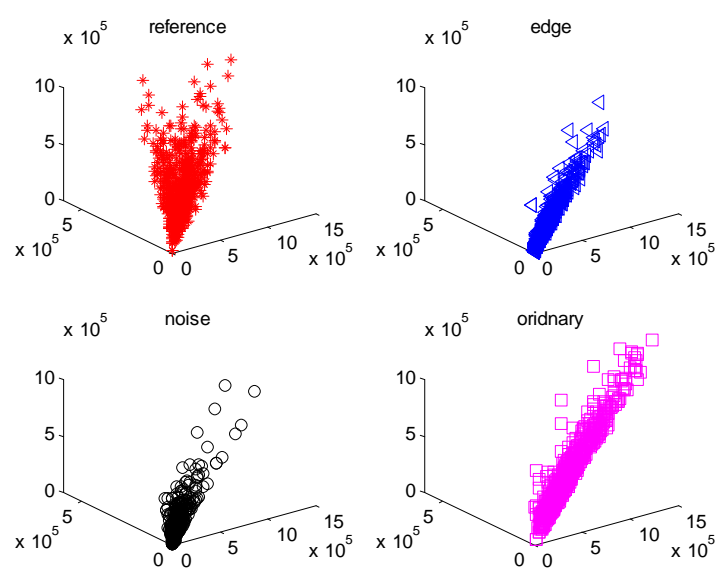

Fig.5. Mean curvature analysis for four kinds of representational sample regions

\section{B. PROPOSED CURVATURE FOR THE FINGERPRINT IMAGE} The fingerprint image is constructed by group of ridges, while the ridges are always not clear and smooth as affected by the background and the noise. The poor quality of ridges interfere the normal curvature calculation correctly. To solve the problem, we proposed a new curvature definition that can satisfy the fingerprint requirement well.

$$
\operatorname{Cur}_{H 2}=\frac{\lambda_{\max }{ }^{2}-\lambda_{\min }{ }^{2}}{\lambda_{\max }{ }^{2}+\lambda_{\min }{ }^{2}}
$$




$$
\operatorname{Cur}_{N}=\frac{\lambda_{\min }}{\lambda_{\max }}-\frac{\lambda_{\max }{ }^{2}-\lambda_{\min }{ }^{2}}{\lambda_{\max }{ }^{2}+\lambda_{\min }{ }^{2}}
$$

As we known $\sqrt{\lambda_{\max }{ }^{2}+\lambda_{\min }{ }^{2}}$ indicate the surface flatness, the proposed new curvature normalizes the square difference of the two eigenvaules with the surface flatness.

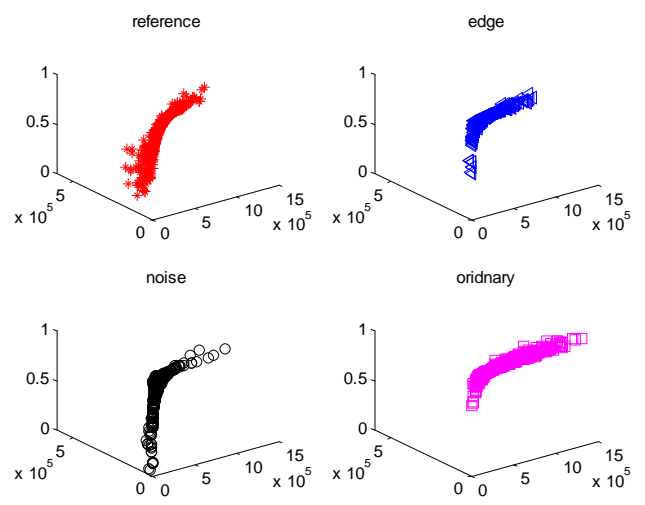

Fig. 6. Hessian2 curvature analysis for four kinds of representational sample regions

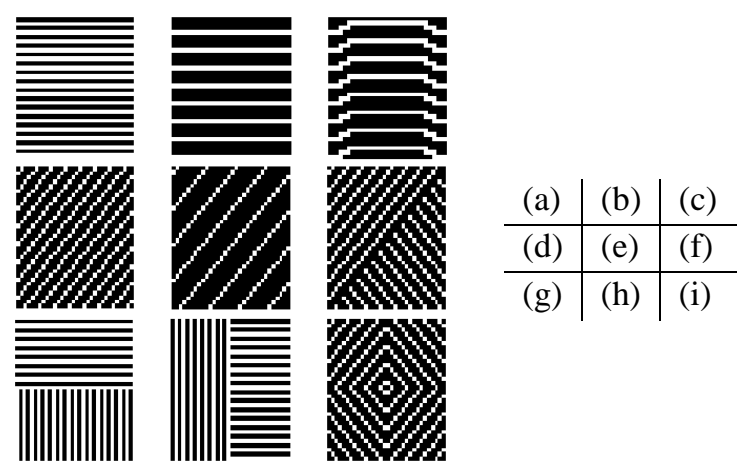

Fig. 7. Nine models of simulated fingerprint ridge

To analysis the curvature properties, we simulate the fingerprint ridges and build nine ridge models as shown in Fig.7. (a-c) are the horizontal ridges with different curves, (d-f) are the diagonal ridges with different ridge distance and curvature changes, (g-h) are the vertical ridges change with different directions, (i) looks like the core point region.

If Gaussian curvature, Mean curvature or Laplacian curvature are used for fingerprint image analysis, they take the ridges minutia so seriously that the model (c) or (d) may be considered as the reference region. Both the Hessian curvature and the proposed curvature can distinguish the global curvature change well. The proposed curvature extend the value range into $[-1,1]$, stronger orientation change region will have the larger value that larger than 0 .

Although the reference point is defined as the point with the maximum curvature value, the method to calculate curvature of all the points and set a point with the biggest curvature one as a reference point is unpractical. Actually a reference point depends heavily on its neighbors' curvature distribution. In the following local analysis, we take four kinds of regions to show their relationship: ordinary region, edge region, noisy region and reference point region.

Further analysis is contributed to 800 different quality images from the database FVC2004_DB1. One ordinary region, edge region, noisy region and reference point region are extracted from each image. Figure 8-12 show Hessian curvature and proposed curvature distribution with different quality image, respectively. We can find the proposed curvature can separate the reference point region with the other regions more efficiently than the Hessian curvature

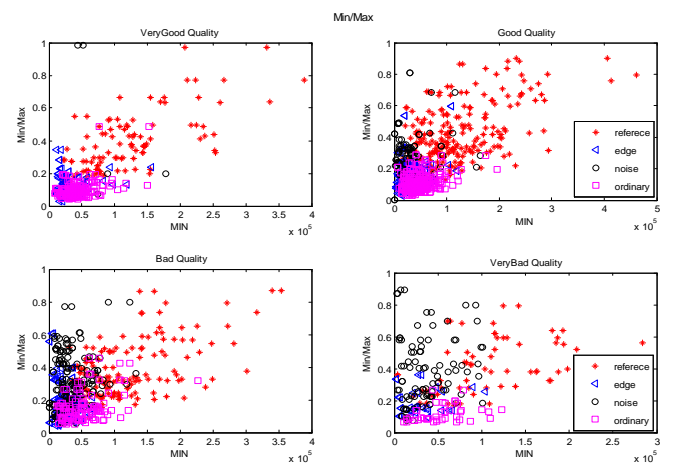

Fig. 8. Analysis of Hessian curvature under different qualities
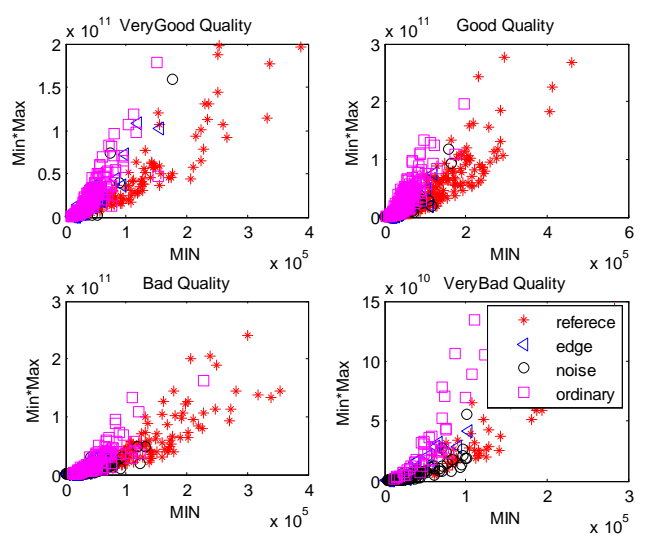

Fig. 9. Analysis of Gaussian curvature under different qualities
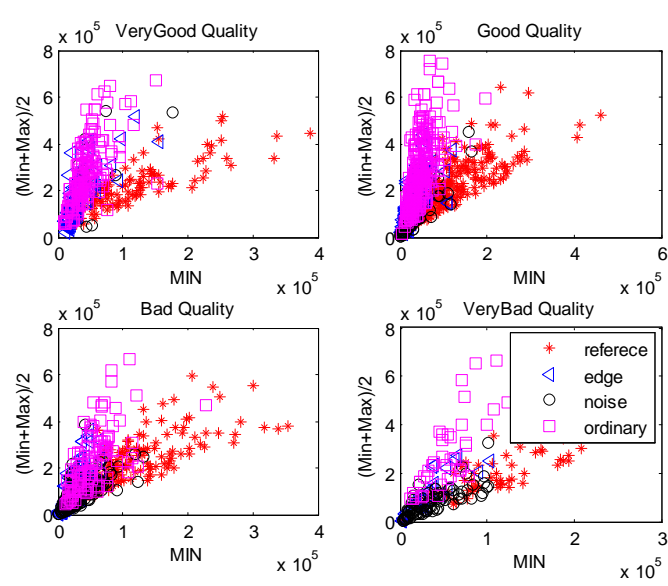

Fig. 10. Analysis of Mean curvature under different qualities 

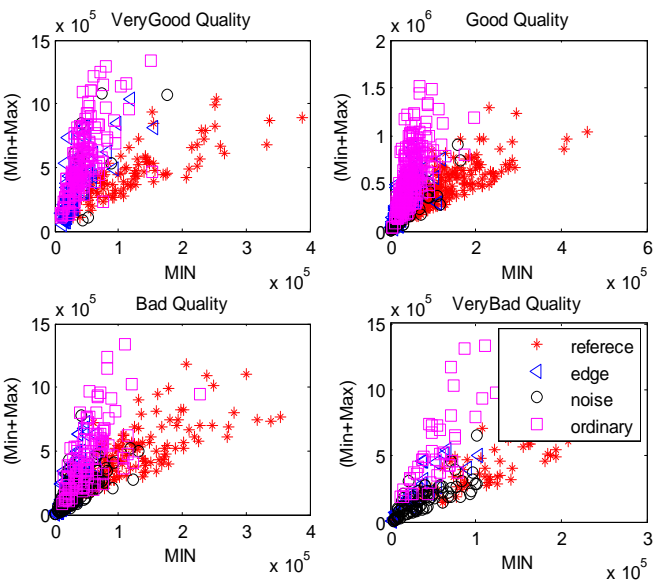

Fig.11. Analysis of Laplacian curvature under different qualities

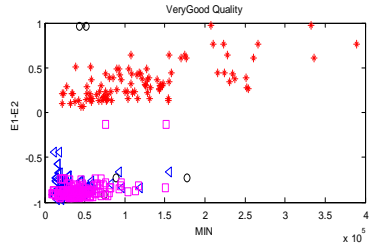

Bad Quali

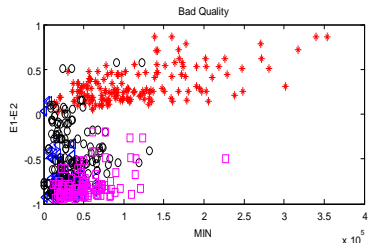

Fig.12. Analysis of Proposed curvature under different qualities

\section{III.REFERENCE POINT LOCALIZATION}

Once a candidate point is determined in the highest level, it is considered as a reference point. The proposed multiresolution determination methods are summarized as following. Fig. 13 shows the procedure of multi-resolution processes.

1. Calculate Coherence1, Coherence 2 of the candidate region at resolution level is $\mathrm{L}$, where $\mathrm{L}$ is initialized by the image size using the following equation.

$$
L=\operatorname{In} \text { teger }\left(\log _{2}(\sqrt{\operatorname{Im} \text { agesize } /(2 \times 2)})\right)
$$

2. Locate the candidate reference regions which satisfy $\operatorname{Cur}_{\mathrm{N}}>0$ and its absolute value is local minimum except when $\operatorname{Cur}_{\mathrm{H}}=\mathrm{Cur}_{\mathrm{H} 2}=0+\delta$ or $1-\delta, \delta$ is a very small value;

3. Calculate the mean and stand deviation values of candidate reference region. And give up the one with the largest standard deviation values.

4. $\mathrm{L}=\mathrm{L}-1$, back to step 1 , until $\mathrm{L}=0$;

5. If there're still several reference region, then choose the one with the smallest standard deviation values. The pixel satisfies condition (2) of the finally located region is set as the position of the reference point.

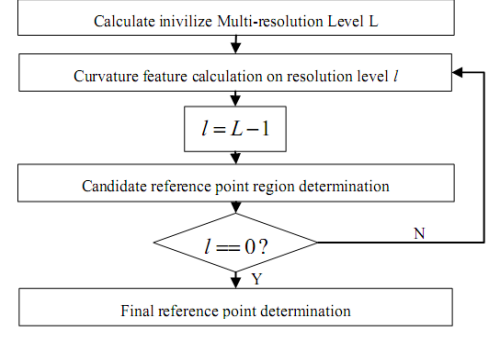

Fig.13. The procedure of fingerprint reference point determination based on multi-Resolution processes

\section{IV.EXPERIMENTAL RESULTS}

\section{A. Database}

The proposed algorithm has been tested on the FVC2004 DB1, DB2 and DB3. Each database consists of 800 fingerprint images from 100 fingers (with 8 images from each finger). DB1 and DB2 were captured by the optical sensor, DB3 was captured by the thermal sensor. The image size is adjusted to $256 \times 256$ pixels and the resolution is 500 dpi. In the databases, there're many poor-quality fingerprints such as partial images with reference points left outside and images with heavy noise like scars, ridge breaks too wet or dry fingerprints, etc. Qualities of the four databases are estimated by optimized orientation certainty method [11], the results are shown in Fig.14. The FVC2004_DB3 databases have the better quality than FVC2004_DB1 and FVC2004_DB2 database, as there are the most Good quality fingerprints in the database FVC2004_DB3.

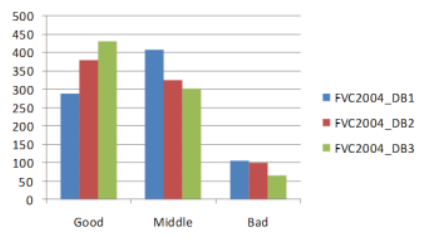

Fig. 14. Quality distribution of the FVC2004

\section{B. FOUR SPECIAL CASES}

In fingerprint reference point determination, we will meet some challenges for four special cases including the multisingular points fingerprint, arch-type fingerprint, and poor quality fingerprint. Some examples for the four cases are shown in Fig. 15-16. There are 14 partial images in the database and their reference points are on the edge or outside of the images. Although it's hard to determine reference points for partial images, our method successfully determine reference points for 4 partial images while Liu's method [2] only determine 2 partial images' reference points. As shown in Fig.7, we can solve the most cases of the partial image and poor quality images. Fig. 8 shows that we did false reference point determination to some bad quality images for its ridges are too faintness. For the partial image reference point is outside of the image, the error can be accepted.

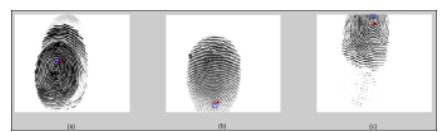

Fig. 15. Examples of the reference points localization for 


\section{International Journal of Advanced Research in Computer and Communication Engineering}

Vol. 5, Issue 6, June 2016

partial image and poor quality image (“ $\Delta$ ", “ $\square$ "denote the reference points detected by our method and the manually located)((a) case of an image with some residue (b) case of an image with a reference point located almost on the edge (c) case of an partial image whose reference point is outside)

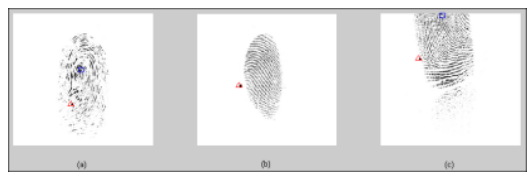

Fig. 16. Examples of False Reference Point Localization

for Partial and Noisy Images (" $\Delta$ " and " $\square$ "denote the reference points detected by the proposed method and the manually-located, respectively.)

\section{ACCURACY AND COMPLEXITY}

The desired position and orientation of the reference point in each fingerprint are not detected previously by experts. To evaluate the performance of our proposed algorithm quantitatively, we manually assigned the desired position of the reference point and the desired reference orientation of each fingerprint. The Euclidean distance between the manually-located reference point and the detected reference point is computed. Fig.17-19 shows the position accuracy of the reference points of FVC2004 DB1, DB2 and DB3. The proposed method is compared with ones based on Mean curvature, Laplacian curvature, Gaussian curvature, Hessian curvature and also the complex filter based method. From the results, the proposed method more precisely determines the reference point location. For FVC2004 DB1, DB2 and DB3, The proposed method shows that the accuracy with smaller than 8 pixels distance error is $89.44 \%, 91.25 \%$ and $91.44 \%$, respectively. And its accuracy within 18 pixels distance error can reach to $94.87 \%, 95.23 \%$ and $95.56 \%$. The proposed method has little effected by fingerprint quality. However, when the fingerprint is arch type with poor quality, our proposed method can't determine its reference point correctly. Besides the advantage of improved accuracy, the proposed method is not time consuming due to the multi-resolution method reduce the determination region step by step.

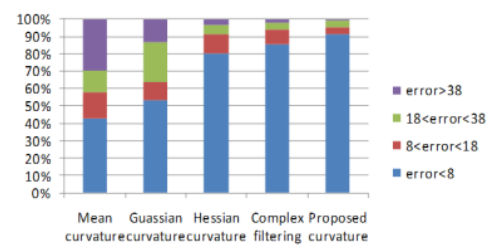

Fig. 17: Reference point determination accuracy for FVC2004_DB1 database

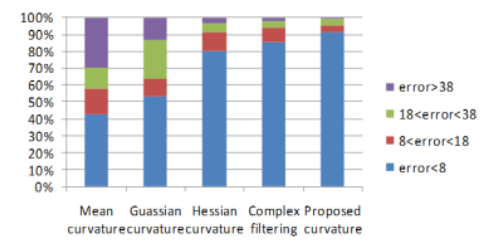

Fig. 18: Reference point determination accuracy for FVC2004_DB2 database

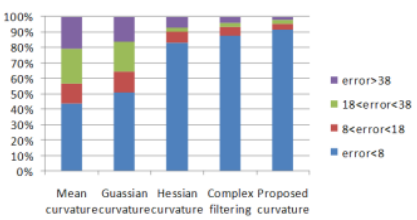

Fig. 19: Reference point determination accuracy for FVC2004_DB3 database

\section{CONCLUSIONS}

In this paper, a new curvature was defined to determine the reference point for the fingerprint. The determination method is based on multi-resolution analysis of the curvature obtained from the relationship of eigenvalues of covariance matrix of image blocks. The proposed curvature can indicate the curvature property of the reference point without the disturbing of noise and background. Experimental results demonstrate that our proposed algorithm can consistently locate a unique reference point with high accuracy for images with different qualities. The located reference point is useful for translational and rotational alignment in fingerprint classification and verification.

\section{REFERENCES}

[1] J. C. Yang, D. S. Park, "A Fingerprint Verification Algorithm Using Tessellated Invariant Moment Features", Neurocomputing, Vol. 71(10-12) pp. 1939-1946, 2008.

[2] M. Liu, X.D. Jiang, A. Kot, "Fingerprint reference-point detection", EURASIPJ. Appl. Signal Process. Vol. 2005 (4), pp: 498-509, 2005

[3] Q. Zhang, H. Yan, "Fingerprint classification based on extraction and analysis of singularities and pseudo ridges, Pattern Recognition ,vol.37 (11),pp:2233-2243,2004

[4] D.J. Li, X.S. Yue, Q.X. Wu, W.X. Kang, "CPGF: Core Point Detection from Global Feature for Fingerprint", Biometric Recognition, Vol. 9428, pp 224-232,2015

[5] R. Doroz, K.Wrobel, M. Palys, "Detecting the Reference Point in Fingerprint Images with the Use of the High Curvature Points". Lecture Notes in Computer Science, 2015, 9012:82-91.

[6] H.K. Lam, Z. Hou, W.Y. Yau, T.P. Chen, "A Systematic Topological Method for Fingerprint Singular Point Detection", ICARCV 2008, pp: 967-972, 2008

[7] A.K. Jain, S. Prabhakar, L. Hong, S. Pankanti, "Filterbank-based fingerprint matching, IEEE Trans. Image Process. Vol. 9 (5), pp:846-859,2000

[8] K. Karu and A. K. Jain, "Fingerprint classification", Pattern Recognition, vol. 29(3), pp:389-404, 1996.

[9] Mohammed S. Khalil, "Reference point detection for camerabased fingerprint image based on wavelet transformation", BIOMEDICAL ENGINEERING ONLINE , vol 14, pp:40, 2015

[10] R. Doroz, K.Wrobel, M. Palys, "Detecting the Reference Point in Fingerprint Images with the Use of the High Curvature Points". Lecture Notes in Computer Science, 2015, 9012:82-91.

[11] S.J. Xie, J.C. Yang, H. Gong, S. Yoon, D.S. Park, Intelligent Fingerprint Quality Analysis using Online Sequential Extreme Learning Machine,Soft Computing,2012,16 (9:1555-1568

[12] S.J. Xie, Y.S. Zhang, "Beam Search Algorithm for Fingerprint Reference Point Determination Based on Joint Orientation Features", International Journal of Science and Research (IJSR), Vol.5(5), pp: 2493 - 2500,2016 